\title{
DNA methylation in the APOE genomic region is associated with cognitive function in African Americans
}

\author{
Jiaxuan Liu', Wei Zhao' ${ }^{1}$ Erin B. Ware ${ }^{2}$, Stephen T. Turner ${ }^{3}$, Thomas H. Mosley ${ }^{4}$ and Jennifer A. Smith ${ }^{1,2^{*}}$
}

\begin{abstract}
Background: Genetic variations in apolipoprotein E (APOE) and proximal genes (PVRL2, TOMM40, and APOC1) are associated with cognitive function and dementia, particularly Alzheimer's disease. Epigenetic mechanisms such as DNA methylation play a central role in the regulation of gene expression. Recent studies have found evidence that DNA methylation may contribute to the pathogenesis of dementia, but its association with cognitive function in populations without dementia remains unclear.

Methods: We assessed DNA methylation levels of $48 \mathrm{CpG}$ sites in the APOE genomic region in peripheral blood leukocytes collected from 289 African Americans (mean age $=67$ years) from the Genetic Epidemiology Network of Arteriopathy (GENOA) study. Using linear regression, we examined the relationship between methylation in the $A P O E$ genomic region and multiple cognitive measures including learning, memory, processing speed, concentration, language and global cognitive function.

Results: We identified eight CPG sites in three genes (PVRL2, TOMM4O, and APOE) that showed an inverse association between methylation level and delayed recall, a measure of memory, after adjusting for age and sex (False Discovery Rate q-value $<0.1$ ). All eight CpGs are located in either CpG islands (CGIs) or CGI shelves, and six of them are in promoter regions. Education and APOE $\varepsilon 4$ carrier status significantly modified the effect of methylation in cg08583001 (PVRL2) and cg22024783 (TOMM40), respectively. Together, methylation of the eight CpGs explained an additional 8.7\% of the variance in delayed recall, after adjustment for age, sex, education, and $A P O E \& 4$ carrier status. Methylation was not significantly associated with any other cognitive measures.

Conclusions: Our results suggest that methylation levels at multiple CpGs in the APOE genomic region are inversely associated with delayed recall during normal cognitive aging, even after accounting for known genetic predictors for cognition. Our findings highlight the important role of epigenetic mechanisms in influencing cognitive performance, and suggest that changes in blood methylation may be an early indicator of individuals at risk for dementia as well as potential targets for intervention in asymptomatic populations.
\end{abstract}

Keywords: DNA methylation, Epigenetics, Cognition, Cognitive function, Delayed recall, Memory, Apolipoprotein E

\footnotetext{
* Correspondence: smjenn@umich.edu

${ }^{1}$ Department of Epidemiology, School of Public Health, University of

Michigan, 1415 Washington Heights, 4602 SPH Tower, Ann Arbor, MI

48109-2029, USA

${ }^{2}$ Survey Research Center, Institute for Social Research, University of Michigan,

Ann Arbor, MI 48104, USA

Full list of author information is available at the end of the article
}

(c) The Author(s). 2018 Open Access This article is distributed under the terms of the Creative Commons Attribution 4.0 International License (http://creativecommons.org/licenses/by/4.0/), which permits unrestricted use, distribution, and reproduction in any medium, provided you give appropriate credit to the original author(s) and the source, provide a link to the Creative Commons license, and indicate if changes were made. The Creative Commons Public Domain Dedication waiver (http://creativecommons.org/publicdomain/zero/1.0/) applies to the data made available in this article, unless otherwise stated. 


\section{Background}

Apolipoprotein E (apoE) plays an essential role in lipid metabolism, and variability in this protein influences several lipid-related diseases of older age, including coronary heart disease, stroke, and Alzheimer's disease (AD) [1]. ApoE also likely influences AD through other biologic mechanisms, including amyloid- $\beta$ aggregation [2]. The APOE $\varepsilon 4$ allele has been identified as the most robust genetic risk factor for $\mathrm{AD}$ and age-related cognitive impairment in multiple populations including African Americans [3]. In addition to the $\varepsilon 4$ allele, other genetic variations in $A P O E$ and genes in close genomic proximity, such as TOMM40, PVRL2, and $A P O C 1$, are also associated with cognition [4-10] and may contribute to the pathogenesis of age-related cognitive impairment.

Cognition and $\mathrm{AD}$ risk may be influenced not only by the DNA sequence but also by epigenetic profiles in the $A P O E$ region. Epigenetic mechanisms, such as histone modification, DNA methylation, and non-coding RNA can regulate gene expression while the underlying DNA sequence remains the same. The epigenome is influenced both by underlying genetic variants as well as by environmental factors including the social environment, health behaviors, and environmental pollutants [11]. Methylation of $\mathrm{CpG}$ dinucleotides, the best understood epigenetic mechanism, is also dynamic over the life course. It is well established that epigenomic patterns of DNA methylation change with age [12]. A recent study in lymphocytes showed that methylation levels at several $\mathrm{CpG}$ sites in $A P O E$ were significantly associated with age, and that these age-related changes in methylation were modified by $A P O E$ genetic variants [13].

Methylation levels at CPG sites in $A P O E$ have also been associated with $\mathrm{AD}$ or its underlying pathology in the brain. For example, decreased methylation was observed in the CpG island (CGI) nested within the fourth exon of $A P O E$ in brain tissue from $\mathrm{AD}$ cases compared to controls [14]. This differential methylation was only observed in brain regions known to impact AD pathophysiology (frontal lobe and hippocampus), suggesting that $A P O E$ methylation may play a role in AD pathogenesis. Further, in postmortem brain tissue, a significant positive association was found between methylation at a separate site within $A P O E$ exon 4 and neuritic amyloid plaque burden, the primary subclinical biomarker of AD pathology [15]. Although these previous studies have explored the relationship between $A P O E$ methylation and $\mathrm{AD}$, there are still many unanswered questions about the mechanistic actions of $A P O E$ methylation and its influences on disease. Additionally, the majority of previous epigenetic studies have focused on participants with European or Asian ancestry [12]. To our knowledge, no study has investigated the association between $A P O E$ methylation and cognitive function in older African Americans without clinicallydiagnosed dementia.

In this study, we investigate the association between peripheral blood leukocyte methylation levels in the $A P O E$ genomic region ( $A P O E, T O M M 40, P V R L 2$, and $A P O C 1)$ and cognitive function in 289 African American participants from the Genetic Epidemiology Network of Arteriopathy (GENOA) study. We also assess whether education and the presence of the APOE \&4 allele confound and/or modify the association between methylation and cognitive function. Deeper insight into the epigenetic mechanisms that influence cognitive function may facilitate improved risk assessment, earlier intervention, and more effective treatment for AD and cognitive decline in the era of precision medicine.

\section{Methods \\ Study sample}

Study participants are African Americans from the Genetic Epidemiology Network of Arteriopathy (GENOA) study, a community-based study of hypertensive sibships designed to investigate the genetics of hypertension and target organ damage in African Americans from Jackson, Mississippi, and non-Hispanic Whites from Rochester, MN $[16,17]$. In the initial phase of GENOA (Phase I: 1996-2001), all members of sibships containing $\geq 2$ individuals with essential hypertension clinically diagnosed before age 60 were invited to participate, including both hypertensive and normotensive siblings. Eighty percent of African Americans $(N=1482)$ from the initial study population returned for the second examination (Phase II: 2001-2005). Hypertension was defined as having systolic blood pressure $\geq 140 \mathrm{mmHg}$, diastolic blood pressure $\geq 90 \mathrm{mmHg}$, or previous diagnosis of hypertension and use of antihypertensive medication. Diabetes was defined as fasting blood glucose $\geq 126 \mathrm{mg} / \mathrm{dL}$ or use of antidiabetic medication. Body mass index (BMI, $\mathrm{kg} / \mathrm{m}^{2}$ ) was calculated from measured weight and height. A subset of participants also had measures of cerebral white matter hyperintensity (WMH). WMH was assessed by Magnetic Resonance Imaging (MRI), and transformed as $\ln (\mathrm{WMH}+1)$ to reduce skewness. In an ancillary study (2001-2006), a total of 1010 African American GENOA participants completed a battery of established cognitive tests that assessed five domains including memory, learning, processing speed, language, and concentration. DNA methylation levels were measured from blood samples collected at Phase I for 422 unrelated participants. Of those with DNA methylation, 289 had at least one cognitive measure and had never been diagnosed with dementia by a physician, and a smaller subset $(N=247)$ also had genotype data available for analysis. 


\section{Cognitive function measures}

Six measures of cognitive function were evaluated [18]: (1) the Wechsler Adult Intelligence Scale Revised Digit Symbol Substitution Task (DSST) assessed processing speed, complex visual attention and visuomotor coordination, (2) the Controlled Oral Word Association Test (COWA, Animal Naming) assessed category fluency, (3) the COWA (FAS) assessed letter fluency, (4) the Rey Auditory Verbal Learning Test (RAVLT) immediate memory trial assessed learning ability, (5) the RAVLT delayed memory trial assessed delayed recall, and (6) the Stroop Color and Word Test (SCWT) assessed concentration effectiveness. For additional details, see Smith et al. [19].

Since global cognitive function is an important indicator of health in older age, reflecting an on-the-spot information processing function employed across various cognitive domains [20], we created a measure of global cognitive function using principal component analysis (PCA) in the sample of GENOA African Americans $(N=1010)$ with cognitive data. The global cognitive function factor was defined as the first unrotated principal component from PCA using the covariance structure of the six cognitive measures described above. This factor explained $53 \%$ of the total variance of the six cognitive measures, and was well loaded by all six measures (loading values ranged from 0.63 to 0.80 ).

\section{DNA methylation measures}

DNA methylation was measured using peripheral blood leukocytes from stored blood samples collected during Phase I. The methylation assay was performed at the Mayo Clinic Advanced Genomics Technology Center using the Illumina Infinium HumanMethylation450 BeadChip. The Minfi $\mathrm{R}$ package [21] was used to preprocess and normalize the data using the SWAN method [22]. $\beta$ values for each $\mathrm{CpG}$ site were calculated as $\beta=\max (\mathrm{M}, 0)$ / $[(\max (U, 0)+\max (M, 0)+100]$, where $M$ represents the fluorescence intensity of the methylated sites and $U$ represents the fluorescence intensity of the unmethylated sites. Individual samples and $\mathrm{CpG}$ sites with a high missing rate (>5\%) were excluded. The proportion of each white blood cell type was estimated using Houseman's method [23]. For each CpG site, the methylation level used for analysis was defined as the $\beta$-value multiplied by 100 (described as a percentage of methylation), adjusted for batch effects and white blood cell proportions. $\beta$-values greater than four standard deviations from the mean of each CpG site were excluded. Forty-eight $\mathrm{CpG}$ sites in the PVRL2TOMM40-APOE-APOC1 genomic region were analyzed. We used Illumina annotation [24] to determine whether each CpG site was in a promoter region (within $1.5 \mathrm{~kb}$ before the transcription start site of a gene) and/or a CGI, CGI shore (0-2 kb from CGI), or CGI shelf (2-4 kb from CGI).

\section{Genotype measures}

We assessed the presence of the APOE $\varepsilon 2$ and $\varepsilon 4$ alleles in a subset of participants with available genotype data $(N=247)$ to evaluate potential confounding and/ or effect modification by $A P O E$ genotype. DNA was isolated using the PureGene DNA Isolation Kit from Gentra Systems (Minneapolis MN). Genotyping, based on polymerase chain reaction amplification techniques, was conducted at the University of Texas-Health Sciences Center at Houston using the TaqMan assay and ABI Prism $^{\odot}$ Sequence Detection System (Applied Biosystems, Foster City CA). Participants were classified as $\varepsilon 2$ carriers (having at least one copy of $\varepsilon 2$ allele) vs. non-carriers, and $\varepsilon 4$ carriers vs. non-carriers.

\section{Statistical analysis}

All statistical analyses were performed in SAS version 9.4 (Cary, NC) and RStudio [25]. Characteristics of the full analysis sample $(N=289)$ and the subset sample with genotype data $(N=247)$ were compared using $\mathrm{T}$ tests for continuous variables or $\chi^{2}$ tests for categorical variables.

The primary analysis was conducted in the full analysis sample $(N=289)$. Linear regression was used to test for association between the methylation level of each $\mathrm{CpG}$ site and each cognitive measure, adjusting for sex and age at cognitive testing (Model 1). A second set of models also included education (less than high school degree, high school degree/GED, beyond high school degree) (Model 2). We assessed models with and without education because adjustment for education can potentially mask the effect of inherited factors on cognition, if they also influence educational attainment [26]. The coMET package [27] was used to create a regional plot for visualization of the association $P$ values. To correct for multiple comparisons, False Discovery Rate (FDR) qvalues [28] were calculated for each cognitive measure across the $48 \mathrm{CpG}$ sites, and FDR $\mathrm{q}<0.1$ was considered significant. For significant associations, models further controlling for hypertension, diabetes, BMI, and current smoking were constructed to test potential confounding by these cognition risk factors. Similarly, in participants with available data for WMH $(N=232)$, we further adjusted the significant associations for hypertension and WMH.

To test the potential confounding effect of the $A P O E$ $\varepsilon 4$ allele on the association between methylation and cognition, a subset analysis was conducted among people with available genotype data $(N=247)$. For Models 1 and 2, $\varepsilon 4$ carrier status was added as an adjustment variable. Only CpG sites showing significant association with cognitive measures in the primary analysis (FDR $\mathrm{q}<0.1)$ were further evaluated in the subset analysis. Associations with $P<0.05$ were considered to remain significant in the subset analysis. 
For cognitive measures that showed significant associations with $\mathrm{CpG}$ sites after accounting for $\varepsilon 4(P<0.05$ in the subset analysis), we quantified the percent variation in the cognitive measure explained by methylation. We first constructed a full model that included all of the significant CpG sites, plus age, sex, and $\varepsilon 4$ carrier status. We then compared this full model to a reduced model that excluded the CpG sites, and calculated the change in $R^{2}$. We repeated this analysis including education in both the full and reduced models.

We also examined education and $\varepsilon 4$ as potential modifiers of the effect of methylation on cognitive function. For the significant $\mathrm{CpG}$ sites in the full sample analysis (FDR $\mathrm{q}<0.1$ ), an interaction term between education and methylation was added to Model 2. Similarly, an interaction term for $\varepsilon 4$ carrier status and methylation was further added to each of the regression models performed in the subset analysis. Interactions with $P<0.05$ were considered significant.

\section{Sensitivity analyses}

People with a previous stroke or prodromal dementia may have cognitive changes or methylation alternations that are distinct from those of non-pathological cognitive aging. We used results from the Mini-Mental State Examination (MMSE), a screening tool for dementia [18], to identify participants that may be in the early stages of dementia. In order to assess whether people with previous stroke or preliminary evidence of dementia influenced the estimated association between methylation and cognition, 46 participants with stroke history and/or with an MMSE score of 23 or less [18] were further excluded from the full sample, and the primary analysis was repeated on the significant $\mathrm{CpG}$ sites in the remaining 243 participants.

Moreover, since the $A P O E \varepsilon 2$ allele has been previously reported to have a protective effect on cognitive function [29], we also assessed whether the $\varepsilon 2$ allele masked the effect of $\varepsilon 4$. To evaluate this, we repeated the subset analysis in 186 participants who were non-carriers of the $\varepsilon 2$ allele. Associations with $P<0.05$ were considered to remain significant in the sensitivity analyses.

\section{Methylation correlations across blood and brain}

To investigate whether cognition-related methylation changes identified in this study (FDR $\mathrm{q}<0.1$ ) may reflect underlying methylation changes in the brain, we used two publically-available databases that compare blood and brain methylation. The Blood Brain DNA Methylation Comparison Tool (http://epigenetics.essex.ac.uk/bloodbrain/) [30] includes methylation levels in whole blood and four brain regions (prefrontal cortex (PFC), entorhinal cortex (EC), superior temporal gyrus (STG), and cerebellum (CER)) in $N=71-75$ matched samples from individuals archived in the MRC London Neurodegenerative Disease Brain Bank. This sample includes both neuropathologically unaffected controls and individuals with variable levels of neuropathology. The Blood-Brain Epigenetic Concordance database (BECon; https://redgar598.shinyapps.io/BECon/) [31] includes methylation levels in blood and three cortical regions (Broadmann area 10 (BA10), prefrontal cortex; Broadmann area 7 (BA7), parietal cortex; and Broadmann area 20 (BA20) temporal cortex) from 16 individuals ranging from 15 to 87 years of age from the Douglas-Bell Canada Brain Bank.

\section{Results}

\section{Sample characteristics}

Sample characteristics are shown in Table 1. The range of age at cognitive testing in the full sample was 45 to 85 years $($ mean $=67$ ), and $71.6 \%$ of the participants were female. Participants were relatively evenly distributed across three education levels. Participant characteristics were not significantly different between the full sample and the subset sample (T-test or $X^{2}$ test $P>0.05$ for all characteristics). The $A P O E \& 4$ allele frequency in the subset sample (0.23) was consistent with previous findings in African Americans [32, 33].

\section{Methylation pattern in the $A P O E$ genomic region}

The mean methylation level (\%) at each CpG site within the PVRL2-TOMM40-APOE-APOC1 region is shown in Fig. 1. The variability in methylation levels across individuals was different between $\mathrm{CpG}$ sites, with standard deviation ranging from 0.7 to $7 \%$. The mean methylation levels of the $13 \mathrm{CpGs}$ in the $A P O E$ gene were consistent with previous observations [13]. Specifically, the first three and the last four CpGs had relatively high methylation (>50\%), while the six CpG sites between them had relatively low methylation $(<50 \%)$. Among all $48 \mathrm{CpG}$ sites in our study, 15 had relatively high methylation (>50\%), and 23 were located in promoter regions. The CpG sites in the promoter regions of PVRL2 and TOMM40 had relatively low methylation $(<50 \%)$, while the mean methylation levels of CpG sites in the $A P O E$ and the $A P O C 1$ promoter regions were more variable. Methylation levels were positively correlated for $82 \%$ of the pairs of CpG sites. For all pairs, Pearson correlation coefficients (r) ranged from -0.15 to 0.59 (Fig. 2).

\section{Association between methylation and cognitive function}

We evaluated the association between DNA methylation of the $48 \mathrm{CpG}$ sites in the PVRL2-TOMM40-APOE$A P O C 1$ genomic region and cognitive measures in the full analysis sample $(N=289)$. We observed significant inverse associations (FDR $\mathrm{q}<0.1$ ) between methylation levels of eight CpG sites and delayed recall (Table 2), 
Table 1 Characteristics of African Americans from the GENOA study with cognitive and DNA methylation data ${ }^{a}$

\begin{tabular}{|c|c|c|c|}
\hline & $\begin{array}{l}\text { Full analysis sample } \\
(N=289)\end{array}$ & $\begin{array}{l}\text { Subset sample } \\
(N=247)\end{array}$ \\
\hline \multicolumn{2}{|l|}{ Age (years) } & $67.2(7.1)$ & $67.4(6.8)$ \\
\hline \multicolumn{2}{|l|}{ Sex (female) } & $207(72 \%)$ & $183(74 \%)$ \\
\hline \multirow[t]{3}{*}{ Education } & Less than high school degree & $95(33 \%)$ & $83(34 \%)$ \\
\hline & High school degree / GED & $81(28 \%)$ & $68(27 \%)$ \\
\hline & Beyond high school degree & $113(39 \%)$ & $96(39 \%)$ \\
\hline \multicolumn{2}{|c|}{ Body mass index (BMl, kg/m²) } & $30.9(6.0)$ & $30.9(6.1)$ \\
\hline \multicolumn{2}{|c|}{ Current smoker } & $34(12 \%)$ & $29(12 \%)$ \\
\hline \multicolumn{2}{|l|}{ Diabetes } & $85(29 \%)$ & $70(28 \%)$ \\
\hline \multicolumn{2}{|c|}{ Hypertension } & $241(83 \%)$ & $205(83 \%)$ \\
\hline \multicolumn{2}{|c|}{ White matter hyperintensity $\left(\mathrm{WMH}, \mathrm{cm}^{3}\right)$} & $12.0(14.2)$ & 11.9 (13.6) \\
\hline \multicolumn{2}{|c|}{ APOE $\varepsilon 4$ carrier } & N/A & $102(41 \%)$ \\
\hline \multicolumn{2}{|c|}{$A P O E$ \&2 carrier } & N/A & $60(24 \%)$ \\
\hline \multicolumn{2}{|c|}{ Processing speed (DSST, symbols) ${ }^{d}$} & $30.6(13.2)$ & $30.4(13.2)$ \\
\hline \multicolumn{2}{|c|}{ Category fluency (Animal Naming, words) ${ }^{d}$} & $14.2(4.4)$ & $14.0(4.2)$ \\
\hline \multicolumn{2}{|c|}{ Letter fluency (FAS, words) ${ }^{d}$} & $28.2(11.5)$ & $28.1(11.5)$ \\
\hline \multicolumn{2}{|c|}{ Learning (RAVLT, words) ${ }^{d}$} & 38. 5 (9.7) & $38.5(9.5)$ \\
\hline \multicolumn{2}{|c|}{ Delayed recall (RAVLT, words) $)^{d}$} & $6.1(3.6)$ & $6.2(3.6)$ \\
\hline \multicolumn{2}{|c|}{ Concentration effectiveness (SCWT, items) ${ }^{d}$} & $20.5(9.1)$ & $20.4(8.9)$ \\
\hline \multicolumn{2}{|c|}{ Global cognitive function } & $-0.12(0.95)$ & $-0.17(0.94)$ \\
\hline
\end{tabular}

${ }^{a}$ Mean (standard deviation) or $\mathrm{n}(\%)$ are presented

${ }^{\mathrm{b}}$ In the full sample, sample sizes for the cognitive measures range from 243 to 289 . A total of 232 participants had data for WMH

'Subset sample consists of subjects with available genotype data

'Values of cognitive scores are the numbers of symbols, words, or items completed within certain time limits as required by each test. For additional details, see Smith et al. [19]

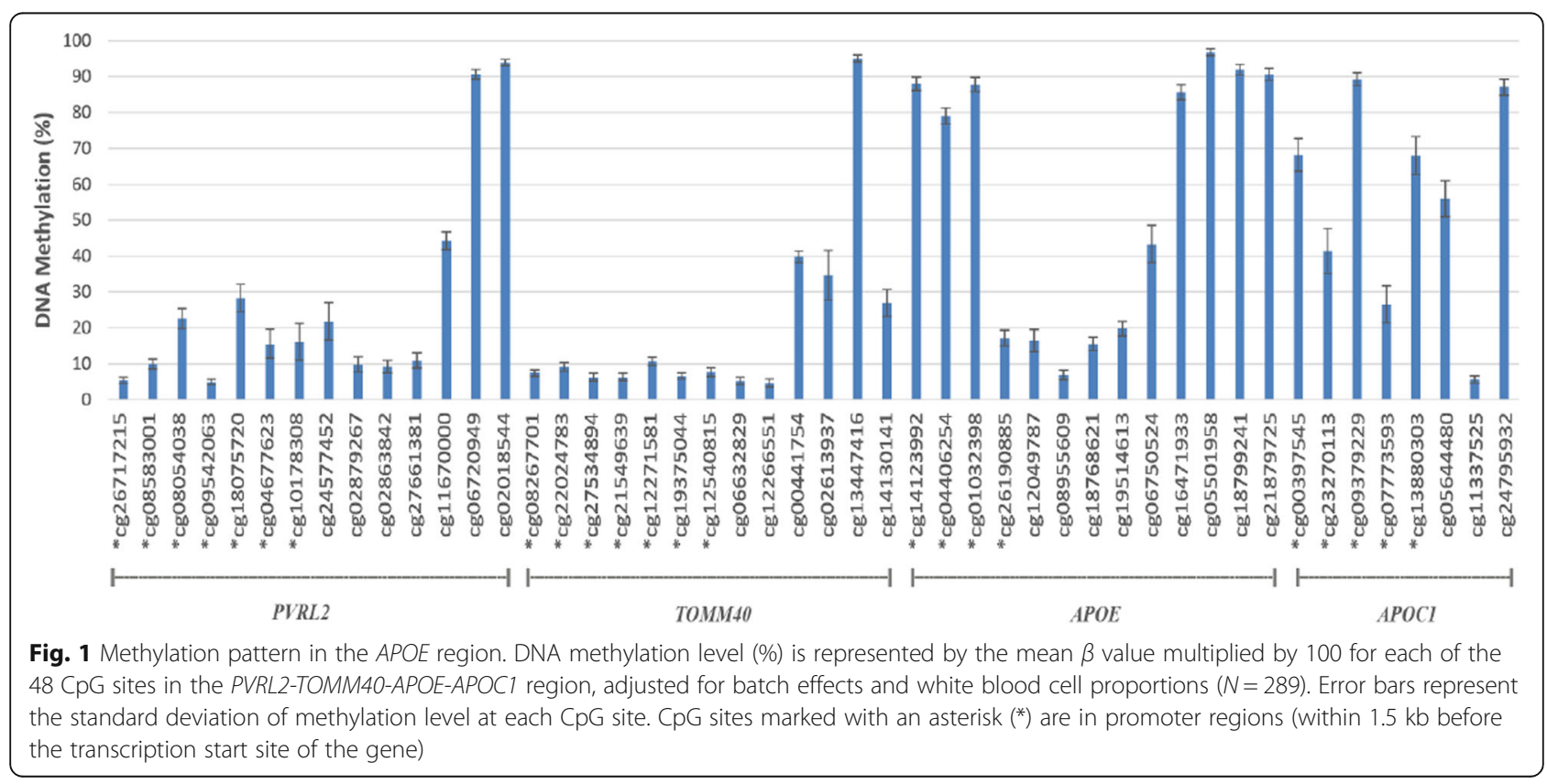




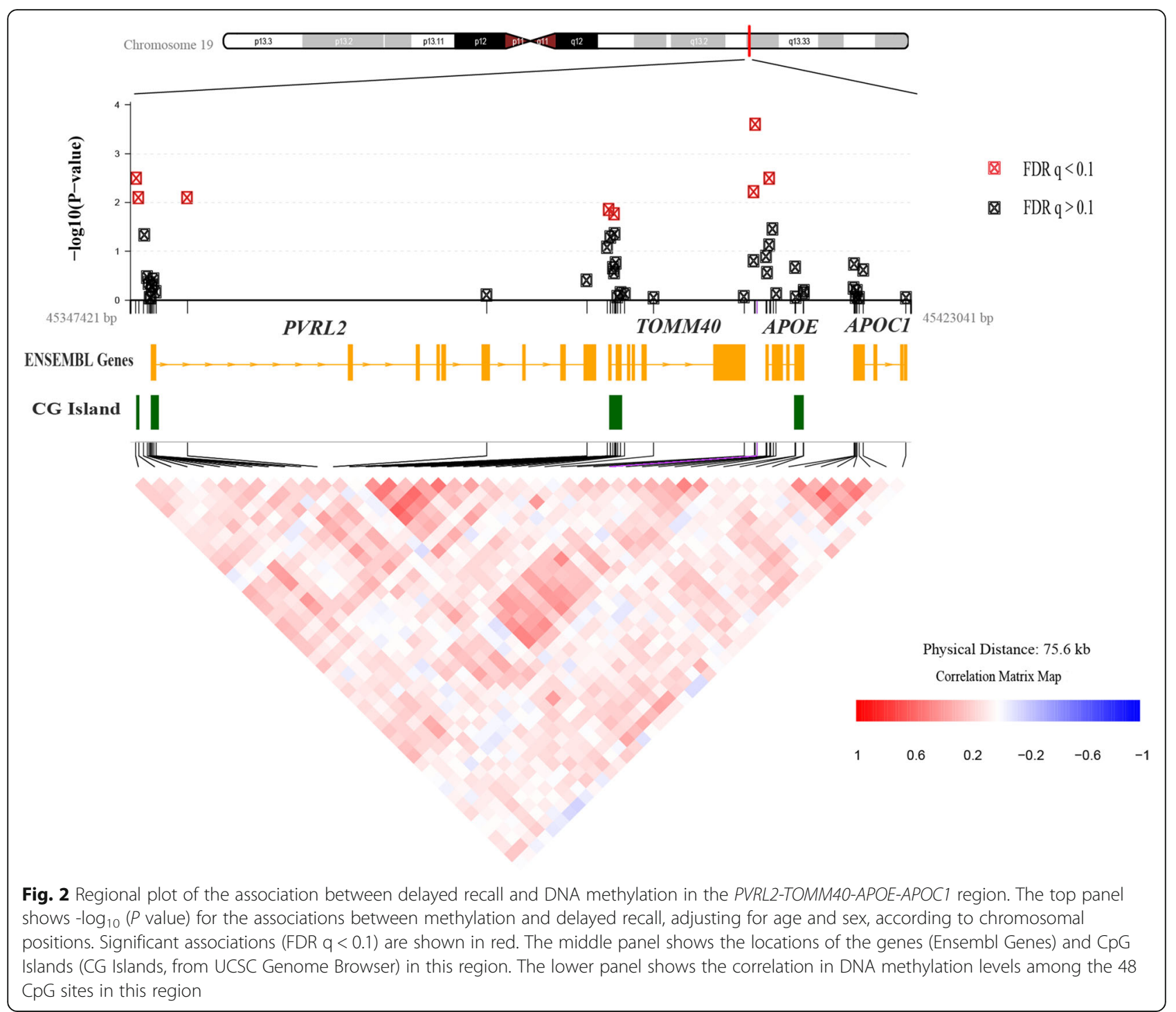

including three CpGs in PVRL2 (cg26717215, cg08583001, and cg11670000), two in TOMM40 (cg22024783 and cg12271581) and three in APOE (cg04406254, cg01032398, and cg18768621). All eight CpGs are located in either CGIs or CGI shelves, and six CpGs are in promoter regions of the three aforementioned genes. The association $P$ values for delayed recall on a $-\log _{10}$ scale according to the chromosomal positions of CpG sites, as well as the correlations among the tested $\mathrm{CpG}$ sites, are shown in a regional plot (Fig. 2). Among the eight $\mathrm{CpG}$ sites, methylation levels of the two CpGs in TOMM4O (cg22024783 and cg12271581) were moderately correlated (Pearson $r=0.55$ ), and both were correlated with APOE cg18768621 (Pearson $r=0.35$ for cg22024783, Pearson $r=0.41$ for cg12271581) and PVRL2 cg11670000 (Pearson $r=0.31$ for cg22024783, Pearson $r=0.30$ for cg12271581). The correlations among the other four CpGs were weak $(r<0.3)$.
After further adjustment for education, the estimated effect of methylation was mildly attenuated for six of the eight CpG sites, except for PVRL2 cg08583001 and TOMM40 cg22024783, where effect estimates increased; six of the eight CpG sites remained significantly associated with delayed recall (FDR $\mathrm{q}<0.1$ ), with the exception of PVRL2 cg11670000 (FDR $\mathrm{q}=0.120$ ) and TOMM40 cg12271581 (FDR q=0.121) (Table 2). All associations remained significant in models further controlling for hypertension and WMH, as well as in models further controlling for hypertension, diabetes, BMI and current smoking $(P<0.05$, data not shown). The methylation levels of the eight $\mathrm{CpG}$ sites were also inversely associated with learning and global cognitive function, adjusted for age, sex, and education; however, the associations were not significant after correction for multiple testing (data not shown). No associations between $\mathrm{CpG}$ methylation 


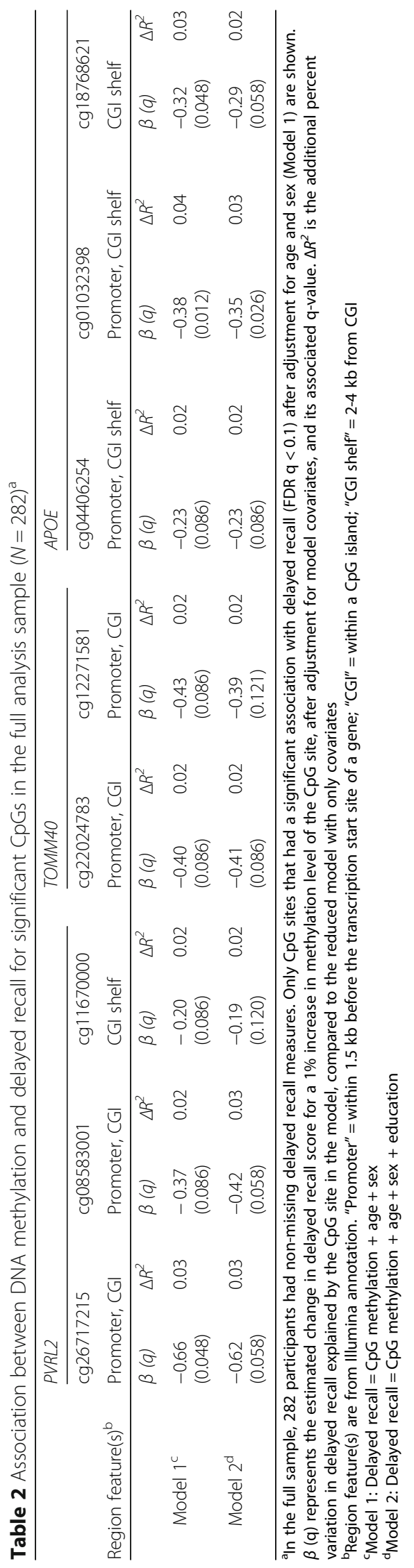


and any of the other cognitive measures were significant after multiple testing correction (data not shown).

When we repeated the analysis for the eight significant CpG sites in the subset sample with available genotypes $(N=242)$, all eight $\mathrm{CpG}$ sites remained significant at $P<0.05$, except for $A P O E \operatorname{cg} 04406254$ with a marginal significance $(P=0.05)$, and effect estimates for some CpGs were slightly attenuated after adjustment for $A P O E$ $\varepsilon 4$ carrier status (Additional file 1: Table S1). Together the eight CpG sites explained an additional 9.1\% of the variance in delayed recall after accounting for age, sex and $A P O E \varepsilon 4$, and $8.7 \%$ when further adjusted for education.

\section{Education and $A P O E$ \&4 carrier status as effect modifiers} A significant interaction was observed between PVRL2 cg08583001 and education on delayed recall ( $P$ for interaction $=0.0046$ ), adjusted for age and sex. Figure 3 shows predicted delayed recall scores calculated for the average age and sex distribution in the full analysis sample $(N=282)$, and compared between the 10th and 90th percentiles of methylation at PVRL2 cg08583001, by education level. The negative effect of increased PVRL2 cg08583001 methylation on delayed recall was only significant in those with education beyond a high school degree. However, for participants with high school degree/GED or less, there was no significant association between methylation and delayed recall, as demonstrated by the overlap between the $95 \%$ confidence intervals of predicted delayed recall scores for the 10th and the 90th percentiles of methylation. This interaction remained significant $(P$ for interaction $<0.05)$ after further controlling for hypertension and $\mathrm{WMH}$, as well as after controlling for hypertension, diabetes, BMI and current smoking (data not shown).

In the subset analysis for individuals with genotype information $(N=242)$, a significant interaction was also observed between TOMM40 $\operatorname{cg} 22024783$ and APOE $\varepsilon 4$ carrier status $(P$ for interaction $=0.013$ ) on delayed recall, adjusted for age and sex. Figure 4 shows predicted delayed recall scores calculated for the average age and sex distribution in the subset sample, and compared between the 10th and 90th percentiles of methylation at PVRL2 TOMM 40 cg22024783, by APOE \&4 carrier status. The negative effect of increased TOMM40 cg22024783 methylation on delayed recall was only observed for $A P O E$ $\varepsilon 4$ carriers, but not for non-carriers. Adjusting for education did not substantively change the results shown in Fig. 4. This interaction remained significant $(P$ for interaction $<0.05$ ) after further controlling for hypertension and $\mathrm{WMH}$, and after further controlling for hypertension, diabetes, BMI and current smoking (data not shown).

\section{Sensitivity analyses}

All eight CpG sites significantly associated in the primary analysis remained at least nominally significantly associated with delayed recall $(P<0.05)$ after excluding people with stroke history or MMSE $\leq 23$ (Additional file 2: Table S2). In the sensitivity analysis that excluded $A P O E \& 2$ allele carriers, results were largely consistent with the analysis that included $\varepsilon 2$ allele carriers, although a few CpGs lost significance due to the reduced sample size (Additional file 3: Table S3). Given the similarity of results before and after excluding those with previous stroke, preliminary evidence of dementia,

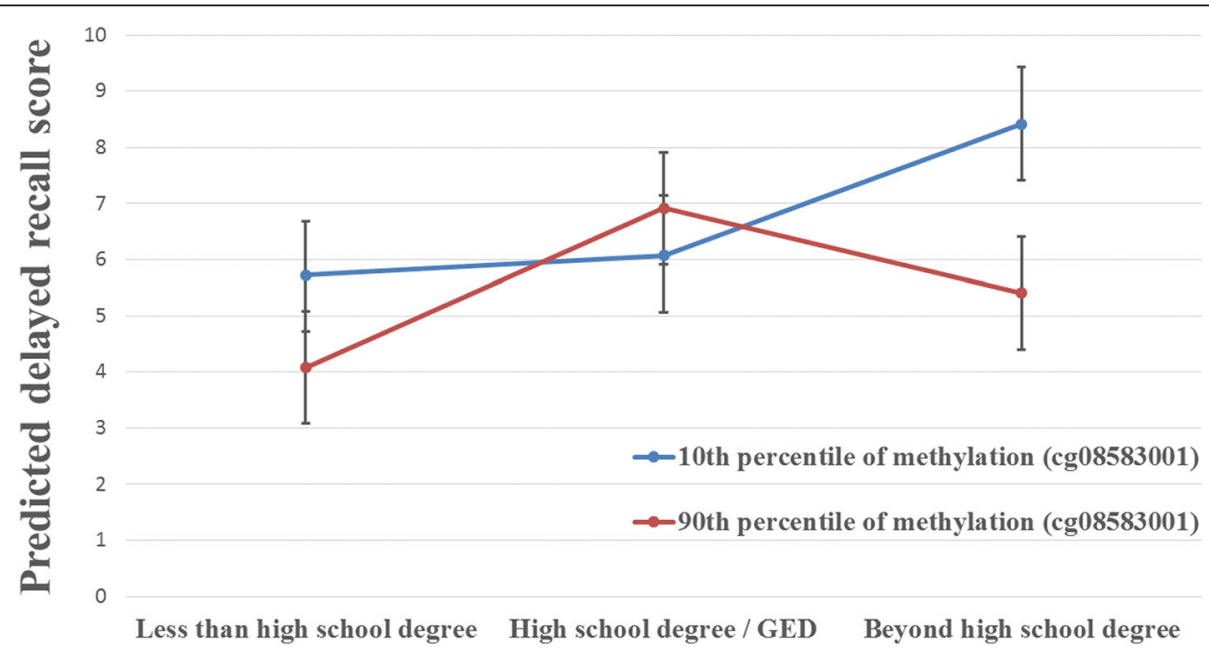

\section{Education}

Fig. 3 Interaction between PVRL2 cg08583001 and education on delayed recall. Predicted delayed recall scores were calculated based on the average age and sex distribution in the full analysis sample $(N=282)$, and compared between the 10th and the 90th percentiles of methylation at PVRL2 cg08583001 by education level. Error bars denote the $95 \%$ confidence interval for each predicted delayed recall score 


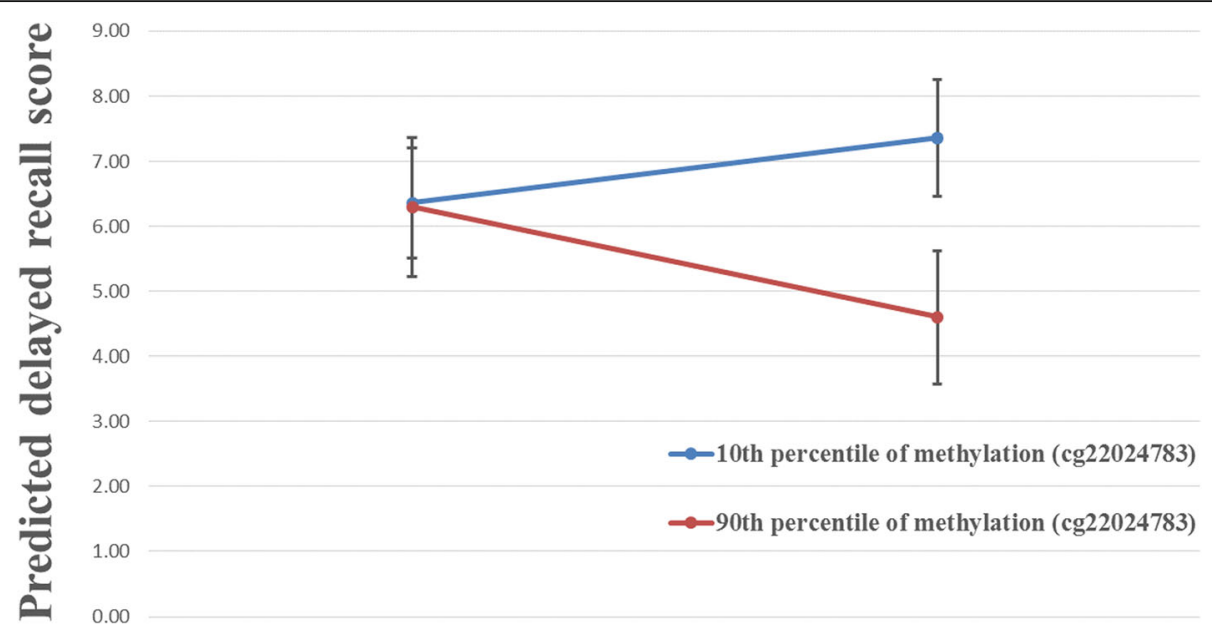

APOE $\varepsilon 4$ non-carrier

APOE $\varepsilon 4$ carrier

Fig. 4 Interaction between TOMM40 cg22024783 and APOE \&4 carrier status on delayed recall. Predicted delayed recall scores were calculated based on the average age and sex distribution in the subset sample $(N=242)$, and compared between the 10th and the 90th percentiles of methylation at TOMM40 cg22024783 by APOE $\varepsilon 4$ carrier status. Error bars denote the $95 \%$ confidence interval for each predicted delayed recall score

and/or the $\varepsilon 2$ allele, it is likely that these factors did not substantively influence the analysis findings.

\section{Methylation correlations across blood and brain}

Correlation between methylation levels in the blood and brain tissue for the eight CpGs associated with delayed recall (FDR q $<0.1$ ) based on data from publically-available databases is shown in Additional file 4: Figure S1 and Additional file 5: Figure S2. Results from both databases show that overall methylation levels are relatively consistent across the tissue types (Additional file 4: Figure S1 and Additional file 5: Figure S2 (panel 1)). That is, CpG sites that are highly methylated ( $>50 \%$ methylation) in blood are also highly methylated in multiple brain regions. Interindividual variations tended to be positively correlated between blood and brain for most of the significant CpG sites evaluated. Correlations between blood and the four evaluated regions using the Blood Brain DNA Methylation Comparison Tool ranged from -0.275 to 0.312 , but 23 of the 32 tested correlations (72\%) were positive (Additional file 4: Figure S1). Using BECon, correlations between blood and the three evaluated regions ranged from -0.56 to 0.45 , but 15 out of the 24 tested correlations (63\%) were positive (Additional file 5: Figure S2 (panel 2)).

\section{Discussion}

While previous studies have suggested that epigenetic modifications may contribute to $\mathrm{AD}[14,15]$, very little is known about the association between DNA methylation and age-related cognitive impairment in those without dementia. To our knowledge this study is the first assessment of the association between DNA methylation and cognitive function in a non-AD population. Studies such as this may enhance understanding of the epigenetic profile for cognitive aging as well as contribute to earlier and novel treatment or intervention strategies for aging-related impairments in cognitive function.

We assessed DNA methylation in the PVRL2-TOMM40$A P O E-A P O C 1$ genomic region, which comprises a moderate to strong linkage disequilibrium (LD) block. Many SNPs in this region, in all four of the genes, have been found to be associated with $\mathrm{AD}$ or cognitive impairment [4-10]. It is still unclear whether the majority of the detected associations between the three neighboring genes and cognitive impairment are independent of $A P O E$ genetic variation or driven at least partially by $\mathrm{LD}$ with $A P O E[5,6]$. Each of the three neighboring genes encodes proteins with biological functions that could plausibly influence cognitive function or AD pathogenesis [9]. Specifically, PVRL2 encodes a cell surface protein involved in the entry activity of herpes simplex virus type 1 (HSV-1) [34, 35]. HSV-1 DNA has been detected in the brains of both healthy elderly and AD cases $[36,37]$, and has been shown to be a risk factor for $\mathrm{AD}$ in $\varepsilon 4$ carriers [38]. TOMM40 encodes a pore subunit of the mitochondrial outer membrane protein translocator, and mitochondrial dysfunction plays an important role in the early pathology of $\mathrm{AD}[39,40]$. APOC1 encodes an apolipoprotein involved in a variety of biological processes including cholesterol metabolism and neuronal apoptosis [41].

Previous functional genomic analysis indicated that genetic variants in this region may have regulatory effects, and gene expression patterns within the region may be modulated by a complex transcriptional 
regulatory structure [42]. The four genes in this region may be mutually influenced by each other through ciselements, supporting the claim that it may be inaccurate to attribute all of this region's genetic influences on $\mathrm{AD}$ and cognition to $A P O E$ alone [8]. We identified eight CpG sites in three genes within this genomic region (PVRL2, TOMM40, and APOE) that had methylation levels significantly associated with delayed recall. Among the eight CpG sites, methylation levels of the two CpGs in TOMM40 (cg22024783 and cg12271581) were moderately correlated, and both were correlated with $A P O E$ cg18768621 and PVRL2 cg11670000. These moderate correlations provide evidence for the underlying coregulation structure in this region, which may be due to underlying patterns of LD as well as shared response to environmental stimuli. The remaining four $\mathrm{CpG}$ sites were only weakly correlated $(r<0.3)$, suggesting that there may also be a role for more site-specific effects on cognition and/or gene expression.

While fewer than half of the 48 CpGs we explored are located in promoter regions, the majority of the significant CpGs (six out of eight) are promoter-nested sites (Table 2), suggesting that the promoter regions of PVRL2, TOMM40, and APOE may have important epigenetic regulatory functions. Also, as shown by the ENCODE regulation track on UCSC Genome Browser (http://genome.ucsc.edu/), signals for common histone marks including H3K4Me3, H3K4Me1, and H3K27Ac have been detected in and around the promoters of these genes, indicating hotspots for histone modification and chromatin remodeling within these regions. Moreover, all of the significant CpG sites are located in either CGIs or CGI shelves (Table 2). However, no significant results were found for APOC1, a gene without a CGI (Fig. 2). Enriched with high CpG content, CGIs have important regulatory function, influencing transcription activity and local chromatin configuration [43]. In addition, previous studies have indicated that CGI flanking regions (both CGI shores and shelves) may also be involved in gene regulation and disease pathophysiology [44, 45]. While most of the CGIs in the human genome are nested in promoter regions, the $A P O E$ gene is among the gene group that does not possess a classical promoter CGI, but a 3'end CGI in the exon region [46]. A previous study showed two regions within this 3'-CGI in which $\mathrm{AD}$ cases had lower methylation levels than controls, and the difference was only significant in brain regions profoundly impacted by $\mathrm{AD}$ pathophysiology (frontal lobe and hippocampus) [14]. In our study, we found three significant CpGs in the shelf of the APOE 3'-CGI, but none in the CGI itself. This is possibly because only three of the $90 \mathrm{CpGs}$ in the APOE 3'-CGI are present on the Illumina 450K chip, and the inter-individual variation within the 3'-CGI has been shown to be low [46]; thus the 48 CpGs sites analyzed in this study may not necessarily represent the other unmeasured sites.

Significant associations (FDR q $<0.1$ ) between methylation and cognitive measures were only observed for delayed recall, but not for any of the other cognitive measures. Nevertheless, the methylation levels of the eight $\mathrm{CpG}$ sites were also consistently inversely associated with learning and global cognitive function, and most of the $P$ values were significant at a nominal level $(P<0.05)$, although they became insignificant after correction for multiple testing. It has been suggested that DNA methylation has a critical role for maintaining normal hippocampal function, and may contribute to age-related changes in synaptic plasticity and memory function [47, 48]. Moreover, as reported in previous population studies on cognitive impairment or dementia, while the APOE $\varepsilon 4$ allele has been associated with various cognitive domains, the strongest association was observed for memory [29]. Our similar findings on methylation in the $A P O E$ region are in accord with previous studies, indicating that the detrimental effects of $A P O E$ and/or other neighboring genes may be more pronounced on memory than other cognitive functions.

The effects of methylation in the APOE genomic region on cognitive function may be mediated by alterations in gene expression. Increased DNA methylation, especially in promoter regions, often results in repressed gene expression. DNA methylation may repress gene expression by either blocking access of transcriptional factors or recruiting methyl-CpG-binding proteins, which can induce further histone modifications and altered chromatin status [49]. In line with this, data from Encyclopedia of DNA Elements (ENCODE) consortium suggested a negative correlation between methylation in the $A P O E$ gene (including the three CpGs showing significance in our study) and gene expression in multiple cell lines [13]. Previous studies have also shown an association between down-regulated gene expression in this region and decreased cognitive function. For example, lower plasma level apoE has been reported to be associated with lower cognitive function [50] and higher risk of dementia [51]. In brain tissue from AD cases, $A P O E$ mRNA level was inversely associated with pathology load, and a separate sample of probable AD cases had a $60 \%$ decrease in $A P O E$ mRNA level in lymphocytes compared to controls [52]. A trend of downregulation of TOMM40 expression was also observed in AD brains as well as blood samples compared with controls [53]. In line with these findings, our study also showed that increased methylation in the promoters of genes in this region is associated with decreased memory function. However, we did not have the ability to assess the relationship between methylation and gene expression levels in this cohort. Future studies on methylation-gene expression and gene expression-cognition associations, in 
populations both with and without $\mathrm{AD}$, will help validate the functional effects of methylation in the $A P O E$ region.

For the majority of the detected associations between methylation and delayed recall, adjustment for education tended to attenuate the estimated effect of methylation, but the changes were small (Table 2). However, we found that education level modified the effect of PVRL2 cg08583001 methylation on delayed recall, such that the deleterious effect of methylation was only present for individuals with education beyond high school. Additionally, we found a significant interaction between TOMM40 cg22024783 and being a carrier of the APOE $\varepsilon 4$ allele. Overall, these findings suggest that the methylation effect is modified by both genetic factors as well as environmental factors. The underlying biological pathways for these interactions will be an important area of future research.

Our study showed that eight CpG sites in the PVRL2TOMM40-APOE-APOC1 genomic region explained nearly $10 \%$ of the variance in the delayed recall measure, after accounting for age, sex, education, and $A P O E \varepsilon 4$. Similarly, a previous epigenome-wide association study (EWAS) reported that 71 significant CpGs together have a higher explanatory power $(28.7 \%)$ for AD pathologic burden than known genetic risk variants (13.9\%) [54]. These findings highlight the important role of epigenetic mechanisms in phenotypic diversity and disease pathophysiology. Future work expanding to other gene regions known to be associated with cognitive impairment, as well as the regions discovered in EWAS studies for AD [3] will improve our understanding of the epigenetic structure of cognitive function.

Our study has several strengths. To our knowledge, this is the first study to assess the association between $A P O E$ methylation and cognitive function in a populationbased cohort of older adults without diagnosed dementia. In addition, our research was conducted in African Americans, an under-studied population with high prevalence of dementia and high allele frequency of $A P O E \varepsilon 4$ [33]. Furthermore, with rich cognitive measures, we were able to assess methylation associations with multiple cognitive domains as well as global cognitive function. Last, while most studies have only focused on the $A P O E$ gene, we also evaluated DNA methylation in the entire LD block surrounding $A P O E$, providing new insights into the epigenetic profiles of this genomic region.

This study also has limitations. First, although the GENOA cohort has substantially more African Americans than previous epigenetic studies of the $A P O E$ region and cognition, the relatively small sample size of participants with both methylation and cognitive measures limited our ability to examine additional regions across the genome. Second, our findings in blood leukocytes provide only limited information on the potential underlying pathological processes in the brain. Recently, it was shown that blood and brain methylation patterns may be quite distinct, and that inferences may not hold across these tissues $[55,56]$. Nevertheless, for the eight significant CpGs detected in our study, consistent methylation patterns were found between blood and brain, and inter-individual variations are positively correlated between blood and brain tissue for the majority of the significant CpGs. While not without limitations, methylation studies of complex neurobiological phenotypes in peripheral tissues are essential for identifying biomarkers that precede dementia and serve as a critical compliment to studies of brain tissue, which can only be obtained after death [30]. Third, our study has limited implications for causality because we did not evaluate longitudinal measures of methylation and cognitive function. Since both methylation and cognition are dynamic over time, further longitudinal studies are necessary to investigate how cognitive function changes with alterations in methylation. Finally, multi-level "omic" approaches that integrate dense genetic, epigenetic, and transcriptomic data from this region will improve our understanding of its genetic influence on cognition and dementia.

\section{Conclusion}

In summary, we identified eight CpG sites in the PVRL2TOMM40-APOE-APOC1 genomic region that showed inverse association between methylation and delayed recall, after accounting for age and sex. Education and $A P O E \varepsilon 4$ modified the effect of methylation at certain CpG sites. Our findings highlight the important role of epigenetic mechanisms in influencing cognitive performance, and suggest that changes in methylation may be fruitful for early identification of individuals at risk for dementia as well as potential targets for intervention in asymptomatic populations.

\section{Additional files}

\begin{abstract}
Additional file 1: Table S1. Association between DNA methylation and delayed recall in the subset sample $(N=242)$. A summary of results for the analysis in subjects with available genotype data, including association coefficients, significance levels, and additional percent variation in delayed recall explained by methylation. (DOC $190 \mathrm{~kb}$ )
\end{abstract}

Additional file 2: Table S2. Sensitivity analysis for the association between DNA methylation and delayed recall in subjects without stroke or dementia $(N=239)$. A summary of results for the sensitivity analysis in subjects without stroke history and/or MMSE $\leq 23$, including association coefficients and significance levels. (DOC $294 \mathrm{~kb}$ )

Additional file 3: Table S3. Sensitivity analysis for the association between methylation and delayed recall in APOE $\varepsilon 2$ non-carriers $(N=183)$. A summary of results for the sensitivity analysis in APOE $\varepsilon 2$ non-carriers, including association coefficients and significance levels. (DOC 185 kb)

Additional file 4: Figure S1. Correlation of blood and brain methylation for the eight CPG sites significantly associated with delayed recall in GENOA: 
Results from the Blood Brain DNA Methylation Comparison Tool. Boxplots of methylation level by tissue type, and scatterplots demonstrating the relationship between methylation in whole blood and four brain regions (prefrontal cortex (PFC), entorhinal cortex (EC), superior temporal gyrus (STG), and cerebellum (CER)) in N=71-75 matched samples from individuals archived in the MRC London Neurodegenerative Disease Brain Bank. Samples from both neuropathologically unaffected controls and individuals with variable levels of neuropathology were included. Plots were generated from the Blood Brain DNA Methylation Comparison Tool (http://epigenetics.essex.ac.uk/bloodbrain/). Only CpG sites that showed a significant association with delayed recall $(F D R q<0.1)$ in the GENOA sample were investigated. (PDF $595 \mathrm{~kb}$ )

Additional file 5: Figure S2. Correlation of blood and brain methylation for the eight $\mathrm{CpG}$ sites significantly associated with delayed recall in GENOA: Results from Blood-Brain Epigenetic Concordance (BECon). Comethylation plots showing the methylation levels in blood and three cortical regions (Broadmann area 10 (BA10), prefrontal cortex; Broadmann area 7 (BA7), parietal cortex; and Broadmann area 20 (BA20) temporal cortex) in 16 individuals ranging from 15 to 87 years of age from the Douglas-Bell Canada Brain Bank (panel 1), as well as a summary of blood-brain correlations (Spearman correlation value), variability of the CpGs (range of beta values between 10th and 90th percentile in the sample), and the effect of cell composition (change in beta value before and after adjustment for cell composition) (panel 2). Plots were generated from the Blood-Brain Epigenetic Concordance database (https://redgar598. shinyapps.io/BECon/. Only CpG sites that showed a significant association with delayed recall (FDR $q<0.1)$ in the GENOA sample were investigated. (PDF $21 \mathrm{~kb})$

\section{Abbreviations}

AD: Alzheimer's disease; APOE: Apolipoprotein E gene; apoE: Apolipoprotein E protein; BMI: Body mass index; CGl: CpG island; COWA: Controlled Oral Word Association Test; DSST: Digit Symbol Substitution Task; EWAS: Epigenomewide association study; FDR: False discovery rate; GENOA: Genetic Epidemiology Network of Arteriopathy; HSV-1: Herpes simplex virus type 1; LD: Linkage disequilibrium; MMSE: Mini-Mental State Examination; PCA: Principal component analysis; RAVLT: Rey Auditory Verbal Learning Test; SCWT: Stroop Color and Word Test; SWAN: Subset-quantile within array normalization

\section{Acknowledgements}

The authors wish to thank the staff and participants of the GENOA study.

\section{Funding}

Support for the Genetic Epidemiology Network of Arteriopathy (GENOA) was provided by the NHLBI (HL054457, HL100185, HL119443, and HL133221) and the NINDS (NS041558) of the NIH.

\section{Availability of data and materials}

The datasets used and/or analyzed in the current study are available from the corresponding author on reasonable request.

\section{Authors' contributions}

$J$ conceived and designed the study jointly with JAS, analyzed the data, and drafted the manuscript. WZ cleaned the DNA methylation data, and both WZ and EBW assisted with data analysis. STT measured the DNA methylation, and THM led the assessment of cognitive function. JAS supervised the study, contributed substantially to manuscript preparation, and provided funding for analysis. All authors were involved in the study design, provided critical revision of the manuscript for intellectual content, and approved the final manuscript.

\section{Ethics approval and consent to participate}

Written informed consent was obtained from all participants, and the University of Michigan Institutional Review Board approved this study (HUM00113791, HUM00008655).

\section{Competing interests}

The authors declare that they have no competing interests.

\section{Publisher's Note}

Springer Nature remains neutral with regard to jurisdictional claims in published maps and institutional affiliations.

\section{Author details}

${ }^{1}$ Department of Epidemiology, School of Public Health, University of Michigan, 1415 Washington Heights, 4602 SPH Tower, Ann Arbor, MI 48109-2029, USA. ${ }^{2}$ Survey Research Center, Institute for Social Research, University of Michigan, Ann Arbor, MI 48104, USA. ${ }^{3}$ Division of Nephrology and Hypertension, Mayo Clinic, Rochester, MN 55905, USA. ${ }^{4}$ Department of Neurology, University of Mississippi Medical Center, Jackson, MS 39126, USA.

Received: 11 October 2017 Accepted: 26 April 2018

Published online: 08 May 2018

\section{References}

1. Eichner JE, Dunn ST, Perveen G, Thompson DM, Stewart KE, Stroehla BC. Apolipoprotein E polymorphism and cardiovascular disease: a HuGE review. Am J Epidemiol. 2002;155(6):487-95.

2. Kim J, Basak JM, Holtzman DM. The role of apolipoprotein E in Alzheimer's disease. Neuron. 2009;63(3):287-303.

3. Smith AR, Mill J, Smith RG, Lunnon K. Elucidating novel dysfunctional pathways in alzheimer's disease by integrating loci identified in genetic and epigenetic studies. Neuroepigenetics. 2016:6:32-50.

4. Bertram L, McQueen MB, Mullin K, Blacker D, Tanzi RE. Systematic metaanalyses of Alzheimer disease genetic association studies: the AlzGene database. Nat Genet. 2007;39(1):17-23.

5. Cervantes S, Samaranch L, Vidal-Taboada JM, Lamet I, Bullido MJ, FrankGarcia A, Coria F, Lleo A, Clarimon J, Lorenzo E, et al. Genetic variation in APOE cluster region and Alzheimer's disease risk. Neurobiol Aging. 2011 32(11):2107.e7-2107.17.

6. Davies G, Harris SE, Reynolds CA, Payton A, Knight HM, Liewald DC, Lopez LM, Luciano M, Gow AJ, Corley J, et al. A genome-wide association study implicates the APOE locus in nonpathological cognitive ageing. Mol Psychiatry. 2014;19(1):76-87.

7. Logue MW, Schu M, Vardarajan BN, Buros J, Green RC, Go RC, Griffith P, Obisesan T.O., Shatz R, Borenstein A, et al. A comprehensive genetic association study of Alzheimer disease in African Americans. Arch Neurol 2011;68(12):1569-1579.

8. Roses A, Sundseth S, Saunders A, Gottschalk W, Burns D, Lutz M. Understanding the genetics of APOE and TOMM40 and role of mitochondrial structure and function in clinical pharmacology of Alzheimer's disease. Alzheimers Dement. 2016;12(6):687-94.

9. Takei N, Miyashita A, Tsukie T, Arai H, Asada T, Imagawa M, Shoji M, Higuchi S, Urakami K, Kimura H, et al. Genetic association study on in and around the APOE in late-onset Alzheimer disease in Japanese. Genomics. 2009;93(5):441-8

10. Caselli RJ, Dueck AC, Huentelman MJ, Lutz MW, Saunders AM, Reiman EM Roses AD. Longitudinal modeling of cognitive aging and the TOMM40 effect. Alzheimers dement. 2012:8(6):490-511. Cacabelos R, Torrellas C. Epigenetics of Aging and Alzheimer's Disease: Implications for Pharmacogenomics and Drug Response. Int J Mol Sci. 2015 Dec 21; 16(12):30483-543

11. Cacabelos R, Torrellas C. Epigenetics of aging and Alzheimer's disease: implications for pharmacogenomics and drug response. Int J Mol Sci. 2015; 16:30483-543.

12. Smith JA, Zagel AL, Sun YV, Dolinoy DC, Peyser PA, Bielak LF, Turner ST, Mosley TH, Kardia SLR. Epigenomic indicators of age in African Americans. Her Gen. 2014:3:137.

13. Ma Y, Smith CE, Lai CQ, Irvin MR, Parnell LD, Lee YC, Pham L, Aslibekyan S, Claas SA, Tsai MY, et al. Genetic variants modify the effect of age on APOE methylation in the genetics of lipid lowering drugs and diet network study. Aging Cell. 2015;14(1):49-59.

14. Foraker J, Millard SP, Leong L, Thomson Z, Chen S, Keene CD, Bekris LM, Yu CE. The APOE gene is differentially methylated in Alzheimer's disease. J Alzheimers Dis. 2015:48(3):745-55.

15. Chibnik LB, Yu L, Eaton ML, Srivastava G, Schneider JA, Kellis M, Bennett DA, De Jager PL. Alzheimer's loci: epigenetic associations and interaction with genetic factors. Ann Clin Transl Neurol. 2015;2(6):636-47.

16. Daniels PR, Kardia SL, Hanis CL, Brown CA, Hutchinson R, Boerwinkle E, Turner ST. Genetic epidemiology network of Arteriopathy study. Familial 
aggregation of hypertension treatment and control in the genetic epidemiology network of Arteriopathy (GENOA) study. Am J Med. 2004; 116(10):676-81.

17. Investigators FBPP. Multi-center genetic study of hypertension: the family blood pressure program (FBPP). Hypertension. 2002;39(1):3-9.

18. Lezak MD. Neuropsychological assessment. New York: Oxford University Press; 1995.

19. Smith JA, Mosley TH, Turner ST, Kardia SLR. In: Agrawal A, editor. Shared genetic effects among measures of cognitive function and leukoaraiosis, Brain injury - pathogenesis, monitoring, recovery and management. Rijeka: InTech; 2012.

20. Davies G, Armstrong N, Bis JC, Bressler J, Chouraki V, Giddaluru S, Hofer E, Ibrahim-Verbaas CA, Kirin M, Lahti J, et al. Genetic contributions to variation in general cognitive function: a meta-analysis of genome-wide association studies in the CHARGE consortium ( $N=53949)$. Mol Psychiatry. 2015;20(2): 183-92.

21. Aryee MJ, Jaffe AE, Corrada-Bravo H, Ladd-Acosta C, Feinberg AP, Hansen KD, Irizarry RA. Minfi: a flexible and comprehensive Bioconductor package for the analysis of Infinium DNA methylation microarrays. Bioinformatics. 2014;30(10):1363-9.

22. Maksimovic J, Gordon L, Oshlack A. SWAN: subset-quantile within array normalization for illumina infinium HumanMethylation450 BeadChips. Genome Biol. 2012;13(6):R44.

23. Houseman EA, Accomando WP, Koestler DC, Christensen BC, Marsit CJ, Nelson $\mathrm{HH}$, Wiencke JK, Kelsey KT. DNA methylation arrays as surrogate measures of cell mixture distribution. BMC Bioinform. 2012;13:86.

24. Bibikova M, Barnes B, Tsan C, Ho V, Klotzle B, Le JM, Delano D, Zhang L, Schroth GP, Gunderson KL, et al. High density DNA methylation array with single CpG site resolution. Genomics. 2011;98(4):288-95.

25. RStudio Team. RStudio: integrated development for R. 2015.

26. Deary IJ, Johnson W. Intelligence and education: causal perceptions drive analytic processes and therefore conclusions. Int J Epidemiol. 2010;39(5):1362-9.

27. Martin TC, Yet I, Tsai PC, Bell JT. coMET: visualisation of regional epigenomewide association scan results and DNA co-methylation patterns. BMC Bioinform. 2015;16:131. https://doi.org/10.1186/s12859-015-0568-2

28. Benjamini Y, Hochberg Y. Controlling the false discovery rate - a practical and powerful approach to multiple testing. J Roy Stat Soc B Met. 1995;57:289-300.

29. Reitz C, Mayeux R. Use of genetic variation as biomarkers for mild cognitive impairment and progression of mild cognitive impairment to dementia. J Alzheimers Dis. 2010;19(1):229-51.

30. Hannon E, Lunnon K, Schalkwyk L, Mill J. Interindividual methylomic variation across blood, cortex, and cerebellum: implications for epigenetic studies of neurological and neuropsychiatric phenotypes. Epigenetics. 2015; 10(11):1024-32.

31. Edgar RD, Jones MJ, Meaney MJ, Turecki G, Kobor MS. BECon: a tool for interpreting DNA methylation findings from blood in the context of brain. Transl Psychiatry. 2017;7(8):e1187

32. Radwan ZH, Wang X, Waqar F, Pirim D, Niemsiri V, Hokanson JE, Hamman RF, Bunker CH, Barmada MM, Demirci FY, et al. Comprehensive evaluation of the association of APOE genetic variation with plasma lipoprotein traits in U.S. whites and African blacks. PLoS One. 2014:9(12):e114618.

33. Tang MX, Maestre G, Tsai WY, Liu XH, Feng L, Chung WY, Chun M, Schofield $P$, Stern Y, Tycko B, et al. Relative risk of Alzheimer disease and age-at-onset distributions, based on APOE genotypes among elderly African Americans, Caucasians, and Hispanics in new York City. Am J Hum Genet. 1996;58(3): 574-84.

34. Warner MS, Geraghty RJ, Martinez WM, Montgomery RI, Whitbeck JC, Xu R, Eisenberg RJ, Cohen GH, Spear PG. A cell surface protein with herpesvirus entry activity (HveB) confers susceptibility to infection by mutants of herpes simplex virus type 1, herpes simplex virus type 2 , and pseudorabies virus. Virology. 1998;246(1):179-89.

35. Martinez WM, Spear PG. Structural features of nectin-2 (HveB) required for herpes simplex virus entry. J Virol. 2001;75(22):11185-95.

36. Gordon L, McQuaid S, Cosby SL. Detection of herpes simplex virus (types 1 and 2) and human herpesvirus 6 DNA in human brain tissue by polymerase chain reaction. Clin Diagn Virol. 1996:6(1):33-40.

37. Lin WR, Wozniak MA, Cooper RJ, Wilcock GK, Itzhaki RF. Herpesviruses in brain and Alzheimer's disease. J Pathol. 2002;197(3):395-402.

38. Itzhaki RF, Lin WR, Shang D, Wilcock GK, Faragher B, Jamieson GA. Herpes simplex virus type 1 in brain and risk of Alzheimer's disease. Lancet. 1997. 349(9047):241-4
39. Devi L, Prabhu BM, Galati DF, Avadhani NG, Anandatheerthavarada HK. Accumulation of amyloid precursor protein in the mitochondrial import channels of human Alzheimer's disease brain is associated with mitochondrial dysfunction. J Neurosci. 2006;26(35):9057-68.

40. Moreira PI, Carvalho C, Zhu X, Smith MA, Perry G. Mitochondrial dysfunction is a trigger of Alzheimer's disease pathophysiology. Biochim Biophys Acta. 2010;1802(1):2-10

41. Leduc V, Jasmin-Belanger S, Poirier J. APOE and cholesterol homeostasis in Alzheimer's disease. Trends Mol Med. 2010:16(10):469-77.

42. Bekris LM, Lutz F, Yu CE. Functional analysis of APOE locus genetic variation implicates regional enhancers in the regulation of both TOMM40 and APOE. J Hum Genet. 2012;57(1):18-25

43. Deaton AM, Bird A. CpG islands and the regulation of transcription. Genes Dev. 2011;25(10):1010-22.

44. Numata S, Ye T, Herman M, Lipska BK. DNA methylation changes in the postmortem dorsolateral prefrontal cortex of patients with schizophrenia. Front Genet. 2014:5:280.

45. Sandoval J, Heyn H, Moran S, Serra-Musach J, Pujana MA, Bibikova M, Esteller M. Validation of a DNA methylation microarray for 450,000 CpG sites in the human genome. Epigenetics. 2011;6(6):692-702.

46. Wang SC, Oelze B, Schumacher A. Age-specific epigenetic drift in late-onset Alzheimer's disease. PLoS One. 2008:3(7):e2698.

47. Penner MR, Roth TL, Barnes CA, Sweatt JD. An epigenetic hypothesis of aging-related cognitive dysfunction. Front Aging Neurosci. 2010;2:9.

48. Xu X. DNA methylation and cognitive aging. Oncotarget. 2015;6(16):13922-32.

49. Mastroeni D, Grover A, Delvaux E, Whiteside C, Coleman PD, Rogers J. Epigenetic mechanisms in Alzheimer's disease. Neurobiol Aging. 2011;32(7): 1161-80.

50. Yasuno F, Tanimukai S, Sasaki M, Hidaka S, Ikejima C, Yamashita F, Kodama C, Mizukami K, Michikawa M, Asada T. Association between cognitive function and plasma lipids of the elderly after controlling for apolipoprotein E genotype. Am J Geriatr Psychiatry. 2012;20(7):574-83

51. Rasmussen $\mathrm{KL}$, Tybjaerg-Hansen A, Nordestgaard BG, Frikke-Schmidt $\mathrm{R}$. Plasma levels of apolipoprotein $\mathrm{E}$ and risk of dementia in the general population. Ann Neurol. 2015;77(2):301-11.

52. Lambert JC, Mann D, Richard F, Tian J, Shi J, Thaker U, Merrot S, Harris J, Frigard $\mathrm{B}$, Iwatsubo $\mathrm{T}$, et al. Is there a relation between APOE expression and brain amyloid load in Alzheimer's disease? J Neurol Neurosurg Psychiatry. 2005;76(7):928-33.

53. Lee TS, Goh L, Chong MS, Chua SM, Chen GB, Feng L, Lim WS, Chan M, Ng TP, Krishnan KR. Downregulation of TOMM40 expression in the blood of Alzheimer disease subjects compared with matched controls. J Psychiatr Res. 2012:46(6):828-30.

54. De Jager PL, Srivastava G, Lunnon K, Burgess J, Schalkwyk LC, Yu L, Eaton ML, Keenan BT, Ernst J, McCabe C, et al. Alzheimer's disease: early alterations in brain DNA methylation at ANK1, BIN1, RHBDF2 and other loci. Nat Neurosci. 2014;17(9):1156-63

55. Yu L, Chibnik LB, Yang J, McCabe C, Xu J, Schneider JA, De Jager PL, Bennett DA. Methylation profiles in peripheral blood CD4+ lymphocytes versus brain: the relation to Alzheimer's disease pathology. Alzheimer's Dementia. 2016;12(9):942-51.

56. Lunnon K, Smith R, Hannon E, De Jager PL, Srivastava G, Volta M, Troakes C, Al-Sarraj S, Burrage J, Macdonald R, Condliffe D. Methylomic profiling implicates cortical deregulation of ANK1 in Alzheimer's disease. Nat Neurosci. 2014;17(9):1164.

Ready to submit your research? Choose BMC and benefit from

- fast, convenient online submission

- thorough peer review by experienced researchers in your field

- rapid publication on acceptance

- support for research data, including large and complex data types

- gold Open Access which fosters wider collaboration and increased citations

- maximum visibility for your research: over $100 \mathrm{M}$ website views per year

At BMC, research is always in progress.

Learn more biomedcentral.com/submissions 